\title{
Análise de Decomposição Estrutural para a Economia Brasileira entre 1995 e 2009
}

\author{
Helena Loiola de Figueiredo ${ }^{1}$ \\ Maria Aparecida Silva Oliveira²
}

Resumo: O objetivo deste artigo é investigar a dinâmica setorial da economia brasileira entre 1995 e 2009 utilizando a decomposição estrutural da matriz insumo-produto. Foram realizadas decomposições do emprego, valor adicionado e valor bruto da produção (VBP). O setor agropecuário apresentou, por um lado, redução no emprego, explicada pelo aumento da produtividade do trabalho, e, por outro crescimento no valor adicionado e no VBP puxado pelas exportações e consumo das famílias. O crescimento no setor industrial foi explicado pelo aumento da demanda final da economia, uma vez que a mudança tecnológica, principalmente a ocorrida no próprio setor industrial, reduziu esse crescimento. Houve um exponencial crescimento do setor de serviços no período, em âmbito do emprego, valor adicionado e VBP.

Palavras-Chave: economia brasileira, decomposição estrutural; análise de insumo-produto.

\begin{abstract}
The objective of this paper is to investigate the sector dynamics of Brazilian economy between 1995 and 2009 using the structural decomposition of the input-output matrix. Decompositions of employment, added value and of gross production value (GPV) were held. The agricultural sector showed, on the one hand, reduction in employment, explained by increase in labor productivity, and on the other a growth of added value and GPV, boosted by exports and household consumption. Growth in the industrial sector has been explained by the increase in final demand of the economy, since technological change, mainly observed in the industry itself, reduced this growth. There was an exponential growth of the service sector in the period, on employment, added value and GPV.
\end{abstract}

1 Mestre em Economia Aplicada pelo Programa de Pós-Graduação em Economia da Universidade Federal de São Carlos (PPGEc - UFSCar)

2 Doutora em Economia Aplicada, Professora do Programa de Pós-Graduação em Economia (PPGEc - UFSCar) 
FIGUEIREDO, H. L. de; OLIVEIRA, M. A. S. Análise de Decomposição Estrutural para a Economia brasileira ...

Keywords: Brazilian economy, structural decomposition; input-output analysis.

JEL: C67; R15

\section{Introdução}

Para que ocorra o desenvolvimento econômico de um país é preciso que uma série de obstáculos sejam superados, é necessário que haja uma mudança estrutural no sentido de garantir que os recursos de uma economia fluam rapidamente para as atividades econômicas modernas, as quais operam com uma maior produtividade econômica (Rodrik, 2013: 2). Trabalhos clássicos como o de Hirschman e Prebisch na década de 1950 abordam o papel da mudança estrutural no crescimento econômico. Como destaca Cimoli (2005: 10), as contribuições teóricas e empíricas do mainstream se concentraram em modelar o crescimento econômico com base em funções de produção agregada, que ignoram as diferenças setoriais, somente após metade dos anos 1980 o tema passou a receber atenção renovada.

Dentro do debate, alguns trabalhos seminais buscaram encontrar padrões de mudança estrutural que pudessem ser aplicados em diferentes momentos do tempo para diferentes economias. Sutcliffe (1971: 33) destaca que esses padrões permitem a análise dos tipos de mudança econômica provenientes da industrialização e sugerem as causas e consequências da divergência entre as economias. Segundo o autor, três categorias de trabalhos se destacam nesse contexto.

A primeira busca identificar padrões na dinâmica de relações entre indústria, agricultura e serviços. Pode-se destacar o estudo de Kuznets (1957) que apontou pioneiramente o fenômeno desigual entre os setores ao longo da trajetória de desenvolvimento das economias: conforme a renda per capita crescia, havia declínio da parcela agrícola no produto nacional e aumento da parcela da indústria.

A segunda categoria detalha os padrões de crescimento dentro do setor industrial. O estudo de Hoffman (apud Sutcliffe, 1971: 33) foi pioneiro nesta categoria, a partir da divisão do produto industrial entre os setores de bens de capital e de consumo, o autor concluiu que a estrutura industrial das economias seguiu um padrão uniforme, independente da dotação de fatores e da tecnologia. No padrão, as atividades produtoras de bens de consumo se desenvolveram primeiro, depois vieram as indústrias de bens de capitais, que se desenvolveram mais rápido que as primeiras.

A terceira categoria, para Sutcliffe (1971: 33), avalia as etapas da industria- 
lização de forma simplificada e dicotômica. De um lado há trabalhos que defendem a especialização produtiva baseada em vantagens comparativas, por outro lado há trabalhos baseados no conceito heterodoxo de que o padrão de especialização importa para o ritmo e alcance do desenvolvimento econômico. Esse debate estimulou estudos empíricos e o estabelecimento de fatos estilizados relevantes sobre o tema. O trabalho de Carvalho e Kupfer (2011) encontra a trajetória de mudança estrutural que vem sendo percorrida pela indústria brasileira. Os autores concluem que a especialização na indústria brasileira se deu em níveis de renda per capita relativamente inferiores aos verificados em outros países. Essa especialização não pode ser vista como causadora do desenvolvimento econômico e dada a rapidez com a qual se deu durante a década de 1990 não é possível eliminar a abertura comercial como um dos catalisadores deste processo.

O processo de abertura comercial da década de 1990 intensificou a necessidade de reestruturação dos setores produtivos da economia. Diante de um novo cenário, em que se passa a enfrentar a concorrência externa abertamente, houve a privatização de diversos segmentos industriais e também a sobrevalorização da taxa real de câmbio entre 1995 e 1998, que estimulou essa perda de participação industrial no PIB. Já em 1999, as mudanças macroeconômicas ${ }^{1}$ (metas de inflação, superávit primário e câmbio flutuante) permitiram um crescimento robusto da produção industrial.

Outra questão que emerge desse processo de abertura comercial é o fenômeno da terceirização e crescimento dos setores de serviços. À medida que um país se desenvolve acumulando um maior nível de renda, o setor de serviço passa a ser o representante de uma maior parcela no PIB. Nesse sentido a indústria perde parte da sua participação no PIB para o setor de serviços. Neste caso, essa mudança estrutural na indústria não é prejudicial à economia porque são mantidos os elos produtivos do setor industrial (Rowthorn E Ramaswamy, 1999). Para o caso brasileiro, Araújo (2010) defende que entre 1995 e 2009 o crescimento econômico ocorre puxado pelo setor de serviços em detrimento do reduzido crescimento dos setores industrial e agropecuário; e destaca que este fato vem contribuindo para a trajetória de crescimento fraca que vêm sendo observada na economia no período recente, já que os empregos gerados no setor de serviços são caracteristicamente de baixa qualidade e de maior precariedade.

Sob a influência dos estudos que buscam identificar os padrões do desenvolvimento econômico segundo a análise setorial e tendo em vista as mudanças na economia brasileira, este trabalho investiga a dinâmica setorial da economia brasileira entre 1995 e 2009, no intuito de distinguir os efeitos do lado da demanda daqueles do lado das mudanças nas ligações entre os setores (denominadas mudanças tecnológicas). A análise é feita para o emprego, o valor adicionado e o valor bruto da produção (VBP).

1 Ver Oreiro e Feijó (2010). 
Foi utilizado o método de decomposição estrutural da análise de insumoproduto proposto por Miller e Blair (2009) que consiste em investigar as fontes de mudança na economia por meio de exercícios de estática comparativa envolvendo as matrizes de insumo-produto. Trabalhos de âmbito nacional e internacional utilizaram esse método para o estudo de mudanças estruturais ocorridas na economia. Franke e Kalmbach (2005) estudaram a mudança estrutural no setor industrial e seus impactos com o setor de serviços para a Alemanha durante a década de 1990. Linden e Dietzenbacher (2000) analisaram os determinantes da mudança estrutural na União Europeia com a aplicação do método RAS para atualizar os coeficientes de uma matriz insumo-produto captando as alterações de efeito-substituição e de efeito-fabricação.

Entre os trabalhos nacionais, Guilhoto et al (2001) compararam a mudança estrutural no Brasil entre 1959 e 1980 com a do Estados Unidos entre 1958 e 1977, a mudança na estrutura das economias foram decompostas entre três componentes dentro e fora do setor (demanda final, tecnologia e as relações intersetoriais). Já Messa (2012) investigou as fontes de mudança estrutural para a economia brasileira ao longo da década de 2000 e comprovou que o menor consumo intermediário de insumos industriais domésticos foi o fator determinante do diferencial de crescimento entre os serviços e a indústria e que a indústria extrativa e o setor agropecuário foram os setores que se destacaram em termos do nível de exportações.

Os coeficientes das matrizes de insumo-produto muitas vezes são interpretados em termos de quantidades físicas, porém tais matrizes são desenvolvidas utilizando-se valores monetários. Para contornar esse problema, Messa (2012) adotou um método de correção para a influência dos preços nos efeitos das mudanças dos coeficientes técnicos sobre o crescimento do produto. Os efeitos, em seu trabalho podem ser interpretados estritamente em termos de quantidade física, refletindo, de fato, mudanças na estrutura produtiva dos respectivos setores. Para este artigo, optou-se pelo deflacionamento das matrizes insumo-produto, ou seja, eliminou-se somente o efeito da variação de preços, os coeficientes continuam refletindo as relações entre valores monetários, porém medidos a preços do ano de referência.

Além do deflacionamento dos dados, outra contribuição desse artigo é examinar a mudança estrutural dos setores da economia brasileira considerando três aspectos distintos, o emprego, o valor adicionado e o VBP através das decomposições dos efeitos da demanda final e dos efeitos da mudança tecnológica, até então nunca realizados para a economia brasileira analisando os três aspectos conjuntamente. Messa (2012) realizou a decomposição estrutural considerando apenas os produtos setoriais. $\mathrm{O}$ artigo ainda vai além do trabalho de Messa (2012), e decompõe os três efeitos da demanda final (nível, mix e composição), permitindo uma análise mais profunda do principal responsável pelo crescimento do emprego, valor adicionado e VBP.

O artigo encontra-se dividido em três seções, além desta introdução. A próxima 
seção apresenta a metodologia. Na segunda seção são apresentados e analisados os resultados empíricos deste trabalho, divididos entre a decomposição da mudança tecnológica e a decomposição da demanda final. A última seção traz algumas considerações finais.

\section{Metodologia}

Os dados utilizados referem-se às matrizes de insumo-produto com agregação de 42 setores, estimadas por Guilhoto e Sesso Filho (2010), essas matrizes estão disponíveis no site do Núcleo de Economia Regional e Urbana da Universidade de São Paulo (NEREUS).

Neste trabalho, as matrizes de 1995 a 2008 foram deflacionadas a preços de 2009 e as mudanças encontradas devem ser empreendidas como reais, dentro do contexto já descrito. Foi utilizado o método "double deflation", que refere-se a um procedimento de dois passos, em que (1) a demanda intermediária, a demanda final e o VBP a preços correntes são deflacionados utilizando um índice de preços por setor construído com base nos dados das tabelas usos e recursos divulgadas nas Contas Nacionais/IBGE; (2) será obtido o índice de preço do valor adicionado que irá balancear a identidade fundamental na qual a soma da coluna deve ser igual a soma da linha no sistema insumo-produto.

Os índices de preços por setor, considerando 1995 como o ano base, para os 42 setores e o período de 1996 a 2009 são:

$\mathrm{Ix}_{1995}=100 \quad, \mathrm{x}=$ setor $1, \ldots$, setor 42

$\mathrm{IX}_{1996}=\mathrm{IX}_{1995}{ }^{*}$ Variação anual de preços do setor $\mathrm{X}_{1996}$

$\mathrm{Ix}_{\mathrm{t}}=\mathrm{Ix}_{\mathrm{t}-1}$ * Variação anual de preços do setor $\quad$, para $\mathrm{t}=1997, \ldots, 2009$

Foi feito um procedimento de mudança do ano base para 2009:

$\mathrm{I}_{\mathrm{t}_{\text {ano-base-2009 }}}=\left(\mathrm{I}_{\mathrm{t}_{\text {ano-base-1995 }}} / \mathrm{I}_{2009_{\text {ano-base-1995 }}}\right) * 100$, para $\mathrm{t}=1995, . ., 2009$

Com os índices já calculados foi possível construir um vetor $\pi^{\mathrm{t}}$ com os índices de preços para os 42 setores da economia brasileira e deflacionar a demanda intermediária $\left(\mathrm{z}^{\mathrm{b}}\right)$, a demanda final $\left(\mathrm{f}^{\mathrm{b}}\right)$, e o $\operatorname{VBP}\left(\mathrm{x}^{\mathrm{b}}\right)$ : 
$\pi^{\mathrm{t}}=\left[\begin{array}{l}\mathrm{I}_{\text {agropecuária }_{\mathrm{t}}} \\ \mathrm{I}_{\text {extrativomineral }_{\mathrm{t}}} \\ \ldots \\ \mathrm{I}_{\text {serviços-privados-não-mercantis }}\end{array}\right], \mathrm{t}=1995, \ldots, 2009$

$$
\begin{aligned}
& \mathrm{z}^{\mathrm{b}}=\hat{\pi}^{\mathrm{t}} \mathrm{z}^{\mathrm{t}} \\
& \mathrm{f}^{\mathrm{b}}=\hat{\pi}^{\mathrm{t}} \mathrm{f}^{\mathrm{t}} \\
& \mathrm{X}^{\mathrm{b}}=\hat{\pi}^{\mathrm{t}} \mathrm{X}^{\mathrm{t}}
\end{aligned}
$$

O segundo passo consiste em calcular o índice de preços que deflacione os dados para o valor adicionado. Pode-se calcular o valor adicionado necessário $\left(\mathrm{v}^{\mathrm{b}}\right)$ para garantir que o VBP permaneça igual tanto na soma das linhas quanto na soma das colunas como,

$$
\left(\mathrm{v}^{\mathrm{b}}\right)^{\prime}=\left(\mathrm{X}^{\mathrm{b}}\right)^{\prime}-\mathrm{i}^{\prime} \mathrm{z}^{\mathrm{b}}
$$

Então, pode-se calcular o deflator do valor adicionado como,

$$
\hat{\mathrm{r}}^{\mathrm{t}}=\hat{\mathrm{v}}^{\mathrm{b}}\left(\hat{\mathrm{v}}^{\mathrm{t}}\right)^{-1} \text {, sendo }\left(\mathrm{v}^{\mathrm{t}}\right) \text { o valor adicionado a preços correntes }
$$

Com as matrizes deflacionadas é preciso definir algumas relações da análise insumo-produto antes de expor os cálculos das decomposições estruturais.

Considerando uma economia desagregada em $\mathrm{n}$ setores; $\mathrm{X}$ um vetor $\mathrm{nx} \mathbf{1}$ de valores brutos de produção setorial; A, uma matriz nxn de coeficientes técnicos; e f , um vetor nx1 de demanda final pelo produto de cada setor. Então, o vetor $X$ de produção setorial pode ser expresso pela equação $X=A X+f$. Após as devidas manipulações algébricas, obtém-se o modelo insumo-produto relacionando os respectivos produtos setoriais:

$$
\mathrm{X}=\mathrm{Lf}
$$

Em que, $\mathrm{L}=(\mathrm{I}-\mathrm{A})^{-1}$, sendo I uma matriz identidade de ordem $\mathrm{n}$. $(\mathrm{I}-\mathrm{A})^{-1}$ é a matriz de coeficientes técnicos de insumos diretos e indiretos, ou a matriz inversa de Leontief, a qual capta os efeitos diretos e indiretos das modificações exógenas da demanda final sobre a produção dos $n$ setores.

As decomposições estruturais realizadas visam estudar as mudanças no VBP, no valor adicionado e no emprego. A seguir, exibe-se como decompor as mudanças no VBP, $\triangle \mathrm{X}$, assumindo que há matrizes insumo-produto para dois períodos (o e 1). 
A mudança no VBP entre os dois anos é:

$$
\Delta \mathrm{X}=\mathrm{X}^{1}-\mathrm{X}^{0}=\mathrm{L}^{1} \mathrm{f}^{1}-\mathrm{L}^{0} \mathrm{f}^{0}
$$

Em que, $\mathrm{f}^{\mathrm{t}}$ é o vetor demanda final no ano $\mathrm{t}$; $\mathrm{L}^{\mathrm{t}}=\left(\mathrm{I}-\mathrm{A}^{\mathrm{t}}\right)^{-1}$ é a matriz de impacto de Leontief no ano $t$.

O método de decomposição estrutural envolve vários exercícios estáticos comparativos nos quais vários coeficientes são mudados, para que se possam comparar os níveis de atividade com um ponto referencial (Miernyk, 1974). Este trabalho utilizou a equação desenvolvida por Dietzenbacher e Los (1998) para a decomposição estrutural do VBP.

$$
\Delta X=\left(\frac{1}{2}\right)(\Delta \mathrm{L})\left(\mathrm{f}^{\mathrm{o}}+\mathrm{f}^{1}\right)+\left(\frac{1}{2}\right)\left(\mathrm{L}^{\mathrm{o}}+\mathrm{L}^{1}\right)(\Delta \mathrm{f})
$$

O primeiro termo do lado direto representa a mudança no VBP se houver uma mudança na tecnologia (implica a mudança na inversa de Leontief - $\Delta \mathrm{L}$ ), enquanto o segundo termo capta o efeito de mudanças da demanda final.

A decomposição da mudança tecnológica é a análise da mudança na matriz A de coeficientes técnicos. Há muitas formas de decomposições de $\Delta \mathrm{A}$, a escolhida por este trabalho foi uma desagregação simples das mudanças nas colunas. Cada coluna da matriz de coeficientes técnicos reflete a produção do setor, identificando as mudanças coluna por coluna é uma forma de perceber os efeitos das mudanças de requerimentos de insumos em cada um dos setores da economia.

Para uma economia com 42 setores,

$$
\mathrm{A}^{1}=\mathrm{A}^{0}+\Delta \mathrm{A}=\left[\begin{array}{ccc}
\mathrm{a}_{1 ; 1}^{\mathrm{o}}+\Delta \mathrm{a}_{1 ; 1} & \cdots & \mathrm{a}_{1 ; 42}^{\mathrm{o}}+\Delta \mathrm{a}_{1 ; 42} \\
\ldots & \cdots & \ldots \\
\mathrm{a}_{42 ; 1}^{\mathrm{o}}+\Delta \mathrm{a}_{42 ; 1} & \cdots & \mathrm{a}_{42 ; 42}^{\mathrm{o}}+\Delta \mathrm{a}_{42 ; 42}
\end{array}\right]
$$

Sendo $\Delta \mathrm{A}^{(\mathrm{j})}=\left[\begin{array}{ccccc}0 & \ldots & \Delta \mathrm{a}_{1 ; \mathrm{j}} & \ldots & \mathrm{0} \\ \ldots & \ldots & \ldots & \ldots & \ldots \\ 0 & \ldots & \Delta \mathrm{a}_{42 ; j} & \ldots & 0\end{array}\right]$ que representa as mudanças tecnológicas do setor $\mathrm{j}$, então 


$$
\Delta \mathrm{A}=\Delta \mathrm{A}^{(1)}+\ldots+\Delta \mathrm{A}^{(\mathrm{j})}+\ldots+\Delta \mathrm{A}^{(42)}=\sum_{\mathrm{j=1}}^{\mathrm{n}} \Delta \mathrm{A}^{\mathrm{j}}
$$

A decomposição da mudança tecnológica $(\triangle \mathrm{A})$ pode ser inserida na equação (12) e assume a seguinte forma,

$$
\Delta \mathrm{X}=\left(\frac{1}{2}\right)\left[\mathrm{L}^{1}\left(\Delta \mathrm{A}^{(1)}\right) \mathrm{L}^{\mathrm{o}}\right]\left(\mathrm{f}^{\mathrm{o}}+\mathrm{f}^{1}\right)+\ldots+\left(\frac{1}{2}\right)\left[\mathrm{L}^{1}\left(\Delta \mathrm{A}^{(42)}\right) \mathrm{L}^{\mathrm{o}}\right]\left(\mathrm{f}^{\mathrm{o}}+\mathrm{f}^{1}\right)
$$

Dessa forma, cada termo da expressão acima representa o efeito da mudança tecnológica para cada setor (j). Se a economia tiver 42 setores, a decomposição da mudança tecnológica terá 42 termos, cada um representando a mudança tecnológica em determinado setor.

Por ser calculado a partir das mudanças na matriz de Leontief, o efeito da mudança tecnológica mostra como variam as ligações entre os setores (enfraquecimento ou fortalecimento dos elos). Os fatores que explicam as mudanças tecnológicas são: inovações; substituição de importações; o aumento dos benefícios decorrentes de economias de escala; as mudanças no mix de produtos (com a adoção de novos substitutos ou de insumos complementares no processo produtivo); a mudança dos preços relativos (dado que os coeficientes técnicos na matriz de Leontief surgem a partir da valoração monetária); e mudanças nos padrões de troca (exportações e também substituição de importações). Esses fatores alteram os coeficientes técnicos na matriz de Leontief e se manifestam no efeito calculado das mudanças tecnológicas (Schuschny, 2005: 63) ${ }^{2}$.

Para a demanda final $(\Delta \mathrm{f})$ alguns fatores podem contribuir para as mudanças observadas entre dois períodos: (1) o valor total de todas as despesas da demanda final (o nível da demanda final); (2) a distribuição da despesa total em todas as categorias da demanda final (chamado efeito composição); (3) o mix de produtos para cada categoria da demanda final. Em um modelo insumo-produto de 42 setores com seis categorias para a demanda final, a matriz da demanda final é definida como,

$\mathrm{F}_{(42 \times 6)}^{\mathrm{t}}=\left[\mathrm{f}_{1}^{\mathrm{t}}, \ldots, \mathrm{f}_{6}^{\mathrm{t}}\right]$, onde $\mathrm{f}_{\mathrm{k}}^{\mathrm{t}}=\left[\begin{array}{c}\mathrm{f}_{1 \mathrm{k}}^{\mathrm{t}} \\ \ldots \\ \mathrm{f}_{42 \mathrm{k}}^{\mathrm{t}}\end{array}\right]$, e é o total de despesas da categoria

2 A decomposição estrutural permite identificar quais atividades apresentaram aumentos no valor bruto da produção pela mudança tecnológica, mas o modelo não contém informações para identificação e análise das suas causas. Ou seja, por este método não é possível verificar qual foi o aumento do produto de um setor pela variação de cada fator que compõe a mudança tecnológica separadamente (inovação, economia de escala, mudança no mix de produto, mudança no preço relativo, mudança no padrão de troca). 
$k$ da demanda final no produto do setor $i$ no ano $t$. O vetor que indica a distribuição de $\mathrm{f}^{t}$ pelas seis categorias da demanda-final (Exportações, Consumo da Administração Pública, Consumo das Instituições Sem Fins de Lucro a Serviço das Famílias (ISFLSFs), Consumo das Famílias, Formação Bruta de Capital Físico (FBCF) e Variação de Estoque), é calculado pela soma das colunas de $\mathrm{F}^{\mathrm{t}}$ dividido por $\mathrm{f}^{\mathrm{t}}$, ou

$$
d_{(6 \times 1)}^{t}=\left[d_{k}^{t}\right]=\left(\frac{1}{f^{t}}\right) y^{t}=\left[\begin{array}{c}
y_{1}^{t} / f^{t} \\
\ldots \\
y_{6}^{t} / f^{t}
\end{array}\right]
$$

Então, $\mathrm{d}_{\mathrm{k}}^{\mathrm{t}}$ representa a proporção da despesa total da demanda-final no ano t que é originária da categoria k. Já a matriz do mix de produção, $\mathbf{B}_{(\mathrm{nxp})}^{\mathrm{t}}$,é

$$
\mathbf{B}^{\mathrm{t}}=\left[\mathbf{b}_{\mathrm{ik}}^{\mathrm{t}}\right]=\left(\mathbf{F}^{\mathrm{t}}\right)\left(\hat{\mathbf{y}}^{\mathrm{t}}\right)^{-1}
$$

A decomposição da mudança da demanda final será,

$$
\begin{aligned}
& \Delta \mathrm{f}=\left(\frac{1}{2}\right)(\Delta \mathrm{f})\left(\mathrm{B}^{\mathrm{o}} \mathrm{d}^{\mathrm{o}}+\mathrm{B}^{1} \mathrm{~d}^{1}\right)+\left(\frac{1}{2}\right)\left[\mathrm{f}^{\mathrm{o}}(\Delta \mathrm{B}) \mathrm{d}^{1}+\mathrm{f}^{1}(\Delta \mathrm{B}) \mathrm{d}^{\mathrm{o}}\right]+ \\
& \left(\frac{1}{2}\right)\left(\mathrm{f}^{\mathrm{o}} \mathrm{B}^{\mathrm{o}}+\mathrm{f}^{1} \mathrm{~B}^{1}\right)(\Delta \mathrm{d})
\end{aligned}
$$

Em que, o primeiro termo capta o efeito nível da demanda-final, o segundo capta o efeito mix da demanda-final, e o terceiro capta o efeito composição da demanda-final.

Para decomposição do emprego, deve-se considerar $\left(\mathrm{e}^{\mathrm{t}}\right)^{\prime}=\left[\mathrm{e}_{\mathrm{o}}^{\mathrm{t}} \ldots \mathrm{e}_{1}^{\mathrm{t}}\right]$ como sendo o vetor de coeficientes de emprego representando a quantidade do trabalho por unidade monetária de produção do setor $i$ no período t. O inverso desses coeficientes representam uma medida de produtividade do trabalho indireta, definidos como:

$$
\mathrm{e}_{\mathrm{i}}^{\mathrm{t}}=\varepsilon_{\mathrm{i}}^{\mathrm{t}} / \mathrm{X}_{\mathrm{i}}^{\mathrm{t}}
$$

Dessa forma, o vetor de emprego setorial no período t será:

$$
\varepsilon^{\mathrm{t}}=\hat{\mathrm{e}}^{\mathrm{t}} \mathrm{X}^{\mathrm{t}}=\hat{\mathrm{e}}^{\mathrm{t}} \mathbf{L}^{\mathrm{t}} \mathrm{f}^{\mathrm{t}}
$$


E o vetor de mudanças no emprego será:

$$
\Delta \varepsilon=\varepsilon^{1}-\varepsilon{ }^{\mathrm{o}}=\hat{\mathrm{e}}^{1} \mathrm{~L}^{1} \mathrm{f}^{1}-\hat{\mathrm{e}}^{\mathrm{o}} \mathrm{L}^{\mathrm{o}} \mathrm{f}^{\mathrm{o}}
$$

Utilizando as mesmas relações empregadas na decomposição da produção, pode-se escrever a equação (21) da seguinte forma:

$$
\begin{aligned}
& \Delta \varepsilon=\left(\frac{1}{2}\right)(\Delta \hat{\mathrm{e}})\left(\mathrm{L}^{1} \mathrm{f}^{1}+\mathrm{L}^{o} \mathrm{f}^{\mathrm{o}}\right)+\left(\frac{1}{2}\right)\left[\hat{\mathrm{e}}^{\mathrm{o}} \Delta \mathrm{Lf} \mathrm{f}^{1}+\hat{\mathrm{e}}^{1} \Delta \mathrm{Lf} \mathrm{f}^{\mathrm{o}}\right]+ \\
& \left(\frac{1}{2}\right)\left(\hat{\mathrm{e}}^{\mathrm{o}} \mathrm{L}^{\mathrm{o}}+\hat{\mathrm{e}}^{1} \mathrm{~L}^{1}\right)(\Delta \mathrm{f})
\end{aligned}
$$

Na equação (22) o primeiro termo é a parcela da variação do emprego devido à mudanças no coeficiente direto de trabalho. $\mathrm{O}$ segundo representa a parcela da variação do emprego setorial devido à transformações tecnológicas. O terceiro capta o efeito da variação da demanda final no emprego setorial.

Finalmente, a decomposição do valor adicionado é semelhante à do emprego. A diferença está em se considerar o vetor de coeficientes direto do valor adicionado, que é representado pela razão entre valor adicionado e o valor da produção $\left(\mathrm{va}_{\mathrm{i}}^{\mathrm{t}}\right)$, ao invés, de se utilizar o vetor de coeficientes direto de emprego. E ainda, foram realizadas as decomposições da demanda final (nível, mix e composição) e da mudança tecnológica para o emprego e valor adicionado.

$$
\begin{aligned}
& \mathrm{va}_{\mathrm{i}}^{\mathrm{t}}=\mathrm{V}_{\mathrm{i}}^{\mathrm{t}} / \mathrm{X}_{\mathrm{i}}^{\mathrm{t}} \\
& \mathrm{V}^{\mathrm{t}}=\widehat{\mathrm{va}}^{\mathrm{t}} \mathrm{X}^{\mathrm{t}}=\widehat{\mathrm{va}}^{\mathrm{t}} \mathrm{L}^{\mathrm{t}} \mathrm{f}^{\mathrm{t}} \\
& \Delta \mathrm{V}=\mathrm{V}^{1}-\mathrm{V}^{0}=\widehat{\mathrm{va}}^{1} \mathrm{~L}^{1} \mathrm{f}^{1}-\widehat{\mathrm{va}}^{0} \mathrm{~L}^{0} \mathrm{f}^{0} \\
& \Delta \mathrm{V}=\left(\frac{1}{2}\right)(\Delta \widehat{\mathrm{va}})\left(\mathrm{L}^{1} \mathrm{f}^{1}+\mathrm{L}^{\mathrm{o}} \mathrm{f}^{\mathrm{o}}\right)+\left(\frac{1}{2}\right)\left[\widehat{\mathrm{va}}^{\mathrm{o}} \Delta \mathrm{Lf}^{1}+\widehat{\mathrm{va}}^{1} \Delta \mathrm{Lf} f^{\mathrm{o}}\right]+ \\
& \left(\frac{1}{2}\right)\left(\widehat{\mathrm{va}}^{\mathrm{o}} \mathrm{L}^{\mathrm{o}}+\widehat{\mathrm{va}}^{1} \mathrm{~L}^{1}\right)(\Delta \mathrm{f})
\end{aligned}
$$




\section{Resultados}

Os resultados para os setores agropecuário, industrial e de serviços foram obtidos por meio da agregação posterior aos cálculos realizados em nível mais desagregado.

A Tabela 1 exibe a variação e o crescimento percentual do emprego, valor adicionado e VBP, entre 1995 e 2009, dos setores agropecuário, industrial, serviços e da economia. Houve um crescimento de $31,41 \%$ do emprego, $61,86 \%$ do valor adicionado e de $72,28 \%$ do VBP da economia brasileira no período. Pode-se notar que as maiores variações e crescimentos do emprego e do valor adicionado foram para o setor de serviços. A agropecuária teve redução no número de postos de trabalho nesse período, porém obteve um crescimento do VBP superior ao da indústria e ao do setor de serviços. A indústria apresentou crescimento do emprego e do valor adicionado superior á agropecuária, porém inferior ao setor de serviços.

TABELA 1 - VARIAÇÃO E CRESCIMENTO DO EMPREGO, VALOR ADICIONADO E VBP ENTRE 1995 E 2009 (MILHÕES DE POSTOS DE TRABALHO E MILHÕES DE REAIS A PREÇOS DE 2009).

\begin{tabular}{lcccccc}
\hline & $\begin{array}{c}\text { Variação } \\
\text { do } \\
\text { emprego }\end{array}$ & $\begin{array}{c}\text { Crescimento } \\
\text { emprego }\end{array}$ & $\begin{array}{c}\text { Variação } \\
\text { Valor } \\
\text { Adicionado }\end{array}$ & $\begin{array}{c}\text { Crescimento } \\
\text { Valor } \\
\text { Adicionado }\end{array}$ & $\begin{array}{c}\text { Variação } \\
\text { do VBP }\end{array}$ & $\begin{array}{c}\text { Crescimento } \\
\text { VBP }\end{array}$ \\
\hline Agropecuária & $-2,32$ & $-12,16 \%$ & 51558,69 & $49,65 \%$ & $\begin{array}{c}125 \\
200,67\end{array}$ & $82,78 \%$ \\
Indústria & 5,31 & $36,54 \%$ & 251736,99 & $52,64 \%$ & $\begin{array}{c}905 \\
503,75\end{array}$ & $65,86 \%$ \\
& & & 747 & $66,95 \%$ & 1268 & $76,65 \%$ \\
Serviços & 20,11 & $50,40 \%$ & 460,96 & 714,14 & $76 \%$ \\
\hline \multirow{2}{*}{ Economia } & 23,10 & \multirow{2}{*}{$31,41 \%$} & 1050 & $61,86 \%$ & 2299 & $72,28 \%$ \\
\hline
\end{tabular}

Fonte: elaborado pela autora, com base nos dados do NEREUS

A Tabela 2 exibe o crescimento percentual, entre 1995 e 2009, do emprego, valor adicionado e VBP das atividades e suas respectivas agregações. Entre as atividades do setor de serviços, o maior crescimento do emprego e do VBP ocorreu para Comunicações e maior crescimento do valor adicionado ocorreu para Serviços Prestados à Família. O menor crescimento do emprego foi para Instituições Financeiras, o menor crescimento do valor adicionado foi para Transportes e o menor crescimento do VBP foi para a Construção Civil no período. 
FIGUEIREDO, H. L. de; OLIVEIRA, M. A. S. Análise de Decomposição Estrutural para a Economia brasileira ...

TABELA 2 - CRESCIMENTO DO EMPREGO, VALOR ADICIONADO E VBP ENTRE 1995 E 2009 POR ATIVIDADE.

\begin{tabular}{|c|c|c|c|c|}
\hline Setor & Atividades & $\begin{array}{l}\text { Crescimento } \\
\text { Emprego }\end{array}$ & $\begin{array}{c}\text { Crescimento } \\
\text { Valor } \\
\text { Adicionado }\end{array}$ & $\begin{array}{c}\text { Crescimento } \\
\text { VBP }\end{array}$ \\
\hline Agro. & Agropecuária & $-12,16 \%$ & $49,65 \%$ & $82,78 \%$ \\
\hline \multirow[t]{4}{*}{ Ind. } & $\begin{array}{c}\text { Indústria } \\
\text { extrativa mineral }\end{array}$ & $21,90 \%$ & $105,98 \%$ & $127,68 \%$ \\
\hline & $\begin{array}{l}\text { Indústria de } \\
\text { otransformação }\end{array}$ & $28,65 \%$ & $41,64 \%$ & $60,72 \%$ \\
\hline & $\begin{array}{c}\text { Produção e } \\
\text { distribuição de } \\
\text { eletricidade, gás } \\
\text { e água }\end{array}$ & $13,89 \%$ & $84,71 \%$ & $102,71 \%$ \\
\hline & Construção Civil & $57,21 \%$ & $53,67 \%$ & $50,94 \%$ \\
\hline \multirow[t]{9}{*}{ Serviços } & Comércio & $44,69 \%$ & $64,97 \%$ & $77,96 \%$ \\
\hline & Transportes & $50,28 \%$ & $41,29 \%$ & $80,18 \%$ \\
\hline & Comunicações & $597,18 \%$ & $95,44 \%$ & $197,91 \%$ \\
\hline & $\begin{array}{l}\text { Instituições } \\
\text { Financeiras }\end{array}$ & $6,04 \%$ & $117,79 \%$ & $94,46 \%$ \\
\hline & $\begin{array}{l}\text { Serviços } \\
\text { Prestados à } \\
\text { Família }\end{array}$ & $49,22 \%$ & $129,59 \%$ & $100,91 \%$ \\
\hline & $\begin{array}{l}\text { Serviços } \\
\text { Prestados à } \\
\text { Empresa }\end{array}$ & $63,50 \%$ & $90,22 \%$ & $87,41 \%$ \\
\hline & $\begin{array}{l}\text { Aluguel de } \\
\text { Imóveis }\end{array}$ & $7,60 \%$ & $48,72 \%$ & $53,86 \%$ \\
\hline & $\begin{array}{l}\text { Administração } \\
\text { Pública }\end{array}$ & $51,04 \%$ & $43,36 \%$ & $49,07 \%$ \\
\hline & $\begin{array}{l}\text { Serviços Privados } \\
\text { não mercantis: } \\
\text { Educação; Saúde } \\
\text { e serviços sociais; } \\
\text { Outros serviços } \\
\text { coletivos, sociais } \\
\text { e pessoais }\end{array}$ & $41,74 \%$ & $62,06 \%$ & $52,88 \%$ \\
\hline
\end{tabular}

Fonte: elaborado pela autora, com base nos dados do NEREUS. 
A Tabela 3 sintetiza a decomposição do crescimento do emprego, valor adicionado e VBP em termos da mudança tecnológica, da demanda final e dos coeficientes diretos (de emprego e de valor adicionado) em termos percentuais. Por exemplo, de acordo com a Tabela 3: o,52\%; $138,41 \%$ e -38,92\% do crescimento do emprego do setor de serviços se devem, respectivamente, as mudanças tecnológicas, da demanda final e do coeficiente direto de emprego.

Importantes conclusões podem ser extraídas a partir da Tabela 3. Em primeiro lugar, nota-se que, para todas as atividades, a mudança na demanda final constituiu o principal fator de crescimento do emprego, valor adicionado e VBP no período, representando mais do que $100 \%$ do crescimento na maioria das atividades.

A agropecuária foi o único setor a apresentar uma variação total do emprego negativa entre os dois anos analisados $(-2,3$ milhões de postos de trabalho, Tabela 1), a mudança tecnológica e a mudança na demanda final contribuíram para a elevação do emprego no período, os sinais negativos desses efeitos, observados na Tabela 3, são devidos ao fato da variação total do emprego desse setor ser negativa. O coeficiente direto de emprego foi o que explicou a perda de empregos na agropecuária. Seu sinal positivo representa o ganho de produtividade que esse setor teve. Esse ganho de produtividade liberou mão-de-obra do setor, o que acabou mais que compensando o aumento do emprego pela mudança tecnológica e da demanda final.

A produtividade do trabalho é medida indiretamente pelo coeficiente direto de emprego. O cálculo do coeficiente recai sobre a relação emprego/produção, se essa relação for menor para o ano de 2009 relativamente ao ano de 1995 a variação do coeficiente será negativa. Por isso, a redução do coeficiente indica ganho de produtividade da mão-de-obra, já que indicará menor emprego em relação a produção ou maior produção em relação ao emprego. Todas as atividades que apresentaram crescimento do emprego no período tiveram o sinal negativo do coeficiente direto de emprego, indicando ganho de produtividade. Apenas as atividades Construção Civil, Comunicações e Administração Pública perderam produtividade da mão-de-obra no período, apresentando valores positivos da variação dos coeficientes diretos emprego.

Houve redução do emprego pela mudança tecnológica para a indústria, ocasionada pelos resultados da indústria extrativa, indústria de transformação e construção civil. A produção e distribuição de eletricidade, gás e água apresentou elevação do emprego pela mudança tecnológica. Já para os serviços, a mudança tecnológica representou $0,52 \%$ do crescimento do emprego desse setor. Houve aumento do emprego pela mudança tecnológica para a maior parte das atividades de serviços (comércio, transportes, comunicações, instituições financeiras, serviços prestados á empresa e aluguel de imóveis), exceto para serviços prestados à família, administração pública e serviços privados não mercantis. 
FIGUEIREDO, H. L. de; OLIVEIRA, M. A. S. Análise de Decomposição Estrutural para a Economia brasileira ...

TABELA 3 - EFEITOS SOBRE O CRESCIMENTO DO EMPREGO, VALOR ADICIONADO E VBP ENTRE 1995 E 2009.

\begin{tabular}{|c|c|c|c|c|c|c|c|c|}
\hline & \multicolumn{3}{|c|}{ EMPREGO } & \multicolumn{3}{|c|}{ VALOR ADICIONADO } & \multicolumn{2}{|c|}{ VBP } \\
\hline SETOR & Tecnológica & $\begin{array}{l}\text { Demanda } \\
\text { final }\end{array}$ & $\begin{array}{c}\text { Coeficiente } \\
\text { direto de } \\
\text { emprego }\end{array}$ & Tecnológica & $\begin{array}{l}\text { Demanda } \\
\text { final }\end{array}$ & $\begin{array}{c}\text { Coeficiente } \\
\text { direto } \\
\text { de valor } \\
\text { adicionado } \\
\end{array}$ & Tecnológica & $\begin{array}{l}\text { Demanda } \\
\text { final }\end{array}$ \\
\hline Agropecuária & $-34,39 \%$ & $-469,44 \%$ & $603,82 \%$ & $11,24 \%$ & $140,38 \%$ & $-51,62 \%$ & $7,64 \%$ & $92,36 \%$ \\
\hline Indústria & $-11,71 \%$ & $128,41 \%$ & $-16,70 \%$ & $-1,13 \%$ & $114,90 \%$ & $-13,77 \%$ & $-0,54 \%$ & $104,34 \%$ \\
\hline Serviços & $0,52 \%$ & $138,41 \%$ & $-38,92 \%$ & $6,06 \%$ & $102,59 \%$ & $-8,65 \%$ & $5,60 \%$ & $94,40 \%$ \\
\hline \multicolumn{9}{|l|}{ ATIVIDADES } \\
\hline Agropecuária & $-34,39 \%$ & $-469,44 \%$ & $603,82 \%$ & $11,24 \%$ & $140,38 \%$ & $-51,62 \%$ & $7,64 \%$ & $92,36 \%$ \\
\hline $\begin{array}{l}\text { Indústria } \\
\text { extrativa } \\
\text { mineral }\end{array}$ & $-17,29 \%$ & $293,17 \%$ & $-175,88 \%$ & $12,94 \%$ & $96,18 \%$ & $-9,12 \%$ & $13,80 \%$ & $86,20 \%$ \\
\hline $\begin{array}{l}\text { Indústria de } \\
\text { transformação }\end{array}$ & $-20,27 \%$ & $128,41 \%$ & $-16,70 \%$ & $-9,20 \%$ & $130,81 \%$ & $-21,61 \%$ & $-4,34 \%$ & $104,34 \%$ \\
\hline $\begin{array}{l}\text { Produção e } \\
\text { distribuição } \\
\text { de } \\
\text { eletricidade, } \\
\text { gás e água }\end{array}$ & $89,25 \%$ & $488,29 \%$ & $-477,54 \%$ & $17,10 \%$ & $98,76 \%$ & $-15,86 \%$ & $14,62 \%$ & $85,38 \%$ \\
\hline $\begin{array}{l}\text { Construção } \\
\text { Civil }\end{array}$ & $-4,51 \%$ & $95,40 \%$ & $9,11 \%$ & $-4,77 \%$ & $100,54 \%$ & $4,23 \%$ & $-4,99 \%$ & $104,99 \%$ \\
\hline Comércio & $7,97 \%$ & $150,18 \%$ & $-58,15 \%$ & $5,80 \%$ & $109,82 \%$ & $-15,62 \%$ & $5,00 \%$ & $95,00 \%$ \\
\hline Transportes & $10,51 \%$ & $135,74 \%$ & $-46,25 \%$ & $12,50 \%$ & $160,74 \%$ & $-73,25 \%$ & $7,10 \%$ & $92,90 \%$ \\
\hline Comunicações & $18,37 \%$ & $36,98 \%$ & $44,65 \%$ & $68,04 \%$ & $103,66 \%$ & $-71,69 \%$ & $37,43 \%$ & $62,57 \%$ \\
\hline $\begin{array}{l}\text { Instituições } \\
\text { Financeiras }\end{array}$ & $134,22 \%$ & $1074,88 \%$ & $-1109,10 \%$ & $8,93 \%$ & $76,07 \%$ & $15,00 \%$ & $10,60 \%$ & $89,40 \%$ \\
\hline $\begin{array}{l}\text { Serviços } \\
\text { Prestados à } \\
\text { Família }\end{array}$ & $-7,28 \%$ & $185,94 \%$ & $-78,66 \%$ & $-3,19 \%$ & $86,62 \%$ & $16,57 \%$ & $-3,90 \%$ & $103,90 \%$ \\
\hline $\begin{array}{l}\text { Serviços } \\
\text { Prestados à } \\
\text { Empresa }\end{array}$ & $0,03 \%$ & $128,84 \%$ & $-28,87 \%$ & $0,03 \%$ & $97,59 \%$ & $2,39 \%$ & $0,03 \%$ & $99,97 \%$ \\
\hline $\begin{array}{l}\text { Aluguel de } \\
\text { Imóveis }\end{array}$ & $11,70 \%$ & $590,12 \%$ & $-501,82 \%$ & $2,09 \%$ & $106,62 \%$ & $-8,71 \%$ & $1,92 \%$ & $98,08 \%$ \\
\hline $\begin{array}{l}\text { Administração } \\
\text { Pública }\end{array}$ & $-2,43 \%$ & $99,20 \%$ & $3,23 \%$ & $-2,80 \%$ & $113,80 \%$ & $-11,00 \%$ & $-2,51 \%$ & $102,51 \%$ \\
\hline
\end{tabular}




\begin{tabular}{|c|c|c|c|c|c|c|c|c|}
\hline & \multicolumn{3}{|c|}{ EMPREGO } & \multicolumn{3}{|c|}{ VALOR ADICIONADO } & \multicolumn{2}{|c|}{ VBP } \\
\hline SETOR & Tecnológica & $\begin{array}{c}\text { Demanda } \\
\text { final }\end{array}$ & $\begin{array}{l}\text { Coeficiente } \\
\text { direto de } \\
\text { emprego }\end{array}$ & Tecnológica & $\begin{array}{l}\text { Demanda } \\
\text { final }\end{array}$ & $\begin{array}{c}\text { Coeficiente } \\
\text { direto } \\
\text { de valor } \\
\text { adicionado }\end{array}$ & Tecnológica & $\begin{array}{c}\text { Demanda } \\
\text { final }\end{array}$ \\
\hline $\begin{array}{l}\text { Serviços } \\
\text { Privados não } \\
\text { mercantis: } \\
\text { Educação; } \\
\text { Saúde e } \\
\text { serviços } \\
\text { sociais; } \\
\text { Outros } \\
\text { serviços } \\
\text { coletivos, } \\
\text { sociais e } \\
\text { pessoais }\end{array}$ & $-16,69 \%$ & $138,78 \%$ & $-22,09 \%$ & $-11,76 \%$ & $99,53 \%$ & $12,23 \%$ & $-13,52 \%$ & $113,52 \%$ \\
\hline
\end{tabular}

Fonte: elaborado pela autora, com base nos dados do NEREUS.

$\mathrm{Na}$ análise do valor adicionado, pela Tabela 3, os crescimentos dos setores agropecuário e de serviços foram explicados tanto pela mudança tecnológica quanto pela mudança na demanda final. A mudança tecnológica apresentou uma contribuição negativa para o crescimento do valor adicionado do setor industrial. E a variação do coeficiente de valor adicionado apontou reduções para todos os três setores, ou seja, houve redução da razão valor adicionado/ valor da produção. Entretanto, essa redução foi menor para serviços, já que instituições financeiras, serviços prestados à família e à empresa apresentaram contribuições positivas do coeficiente direto de valor adicionado.

O crescimento do VBP, assim como os demais, foi explicado principalmente pela mudança na demanda final. A mudança tecnológica contribuiu para esse crescimento no setor agropecuário e de serviços; e reduziu o VBP da indústria (as contribuições negativas da mudança tecnológica da indústria de transformação e construção civil colaboraram para esse resultado).

\subsection{Decomposição da Mudança Tecnológica}

A Tabela 4 ilustra quanto às mudanças tecnológicas ocorridas nos setores indutores influenciaram o crescimento do emprego, do valor adicionado e do VBP nos setores sob efeito. Por exemplo, de acordo com a Tabela 4, 1,99\% do crescimento do emprego no setor de serviços foi devido à mudança tecnológica 
ocorrida na indústria, $0,51 \%$ pela mudança tecnológica da agropecuária e $-1,98 \%$ pela mudança tecnológica de serviços. Ou seja, as mudanças tecnológicas ocorridas no próprio setor frearam o crescimento do emprego.

Lembre-se que, para o caso da agropecuária houve redução do emprego então, apesar de apresentarem sinais negativos, as mudanças tecnológicas ocorridas na agropecuária e indústria aumentaram o emprego na agropecuária. Já as mudanças tecnológicas em serviços reduziram o emprego da agropecuária. A indústria sofreu aumento do emprego pelas mudanças tecnológicas da agropecuária (em $0,13 \%$ ) e redução pelas mudanças dos setores industriais (em -8\%) e de serviços (em -3,85\%).

TABELA 4 - DECOMPOSIÇÃO DO EFEITO TECNOLÓGICO

\begin{tabular}{lcccc}
\hline \multirow{4}{*}{ EMPREGO } & \multicolumn{4}{c}{ Setor sob efeito } \\
\cline { 2 - 5 } & Setor Indutor & Agropecuária & Indústria & Serviços \\
\hline & Agropecuária & $-23,90 \%$ & $0,13 \%$ & $0,51 \%$ \\
& Indústria & $-23,92 \%$ & $-8,00 \%$ & $1,99 \%$ \\
VALOR & Serviços & $13,43 \%$ & $-3,85 \%$ & $-1,98 \%$ \\
ADICIONADO & Agropecuária & $6,73 \%$ & $2,78 \%$ & $0,35 \%$ \\
& Indústria & $8,24 \%$ & $-5,16 \%$ & $2,30 \%$ \\
VALOR & Serviços & $-3,73 \%$ & $1,26 \%$ & $3,40 \%$ \\
BRUTO DE & Agropecuária & $4,32 \%$ & $2,25 \%$ & $0,30 \%$ \\
PRODUÇÃO & Indústria & $5,71 \%$ & $-4,76 \%$ & $2,09 \%$ \\
(VBP) & Serviços & $-2,38 \%$ & $1,97 \%$ & $3,21 \%$ \\
\hline
\end{tabular}

Fonte: elaborado pela autora, com base nos dados do NEREUS.

Três conclusões podem ser estabelecidas a partir da Tabela 4. Em primeiro lugar, para a agropecuária, as mudanças tecnológicas ocorridas no setor de serviços ampliaram a redução do emprego e reduziram o crescimento do valor adicionado e do VBP. Em segundo lugar, para a indústria, as mudanças tecnológicas ocorridas no próprio setor foram as que mais reduziram o crescimento do emprego, valor adicionado e VBP. E em terceiro lugar, para serviços, a mudança tecnológica ocorrida no setor industrial foi a que mais colaborou para o crescimento do emprego, já as mudanças tecnológicas ocorridas no próprio setor de serviços foram as que mais colaboraram com os crescimentos do valor adicionado e do VBP do setor.

A Tabela 5 ilustra os resultados desagregados para as atividades dos setores. Por exemplo, segundo a Tabela 4, as mudanças tecnológicas ocorridas no 
setor industrial foram as que mais reduziram os crescimentos do emprego, valor adicionado e VBP no próprio setor. Assim, de acordo com a Tabela 5, as atividades Indústria Extrativa Mineral; Indústria de Transformação; e Construção Civil foram as que sofreram as reduções desses crescimentos. Especialmente a Indústria de Transformação que foi a atividade que mais sofreu o efeito das mudanças tecnológicas industriais no crescimento do emprego, do valor adicionado e do VBP, apresentando reduções de 12,89\%, $30,94 \%$ e $41,49 \%$, respectivamente.

Em relação às atividades do setor de serviços, Transportes e Comunicações foram as que tiveram maiores crescimentos do emprego, do valor adicionado e do VBP pelas mudanças tecnológicas do setor de serviços. Comércio, Instituições Financeiras, Serviços Prestados à Empresa e Aluguel de Imóveis foram as que apresentaram maiores crescimentos do emprego, valor adicionado e VBP pela mudança tecnológica ocorrida no setor industrial. Serviços prestados à Família tiveram os maiores crescimentos pela mudança tecnológica ocorrida na agropecuária. Com destaque, a atividade Comunicações apresentou o maior crescimento do valor adicionado e VBP causado pela mudança tecnológica do setor de serviços, $63,75 \%$ e 42,53\%, respectivamente. $O$ fato que pode ajudar a explicar o motivo da mudança tecnológica ter a maior contribuição para o setor de Comunicações é o elevado fluxo de Investimento Direto Externo (IDE) para a infra-estrutura desse setor na metade década de noventa, principalmente no caso das telecomunicações, observado no estudo de Sarti e Laplane (2002: 83-4):

Esse grupo recebeu elevados investimentos de novas empresas estrangeiras, em especial nos setores de Informática e Equipamentos de Telecomunicações, em grande parte associados à instalação e operação de novas plantas no país. Os investimentos foram realizados quase exclusivamente por empresas produtoras de bens finais (montadoras). Não houve investimentos significativos de produtores de insumos e componentes para os bens finais. 
TABELA 5 - DECOMPOSIÇÃO DESAGREGADA DO EFEITO TECNOLÓGICO

\begin{tabular}{|c|c|c|c|}
\hline \multirow{3}{*}{ Atividade sob efeito } & \multicolumn{3}{|c|}{ EMPREGO } \\
\hline & \multicolumn{3}{|c|}{ Setor indutor } \\
\hline & Agropecuária & Indústria & Serviços \\
\hline Agropecuária & $-23,90 \%$ & $-23,92 \%$ & $13,43 \%$ \\
\hline Indústria extrativa mineral & $-1,63 \%$ & $-12,53 \%$ & $-3,12 \%$ \\
\hline Indústria de transformação & $0,27 \%$ & $-12,89 \%$ & $-7,66 \%$ \\
\hline $\begin{array}{l}\text { Produção e distribuição de } \\
\text { eletricidade, gás e água }\end{array}$ & $2,39 \%$ & $26,43 \%$ & $60,44 \%$ \\
\hline Construção Civil & $0,06 \%$ & $-3,33 \%$ & $-1,25 \%$ \\
\hline Comércio & $1,79 \%$ & $4,75 \%$ & $1,42 \%$ \\
\hline Transportes & $0,15 \%$ & $1,65 \%$ & $8,71 \%$ \\
\hline Comunicações & $0,07 \%$ & $-0,77 \%$ & $19,07 \%$ \\
\hline Instituições Financeiras & $4,36 \%$ & $79,77 \%$ & $50,09 \%$ \\
\hline Serviços Prestados à Familía & $0,10 \%$ & $0,03 \%$ & $-7,41 \%$ \\
\hline Serviços Prestados à Empresa & $0,45 \%$ & $14,12 \%$ & $-14,54 \%$ \\
\hline Aluguel de Imóveis & $1,54 \%$ & $20,69 \%$ & $-10,54 \%$ \\
\hline Administração Pública & $-0,02 \%$ & $-0,79 \%$ & $-1,62 \%$ \\
\hline $\begin{array}{l}\text { Serviços Privados não-mercantis: } \\
\text { Educação; Saúde e serviços sociais; } \\
\text { Outros serviços coletivos, sociais e } \\
\text { pessoais }\end{array}$ & $-0,19 \%$ & $-6,75 \%$ & $-9,75 \%$ \\
\hline \multirow{3}{*}{ Atividade sob efeito } & \multicolumn{3}{|c|}{ VALOR ADICIONADO } \\
\hline & \multicolumn{3}{|c|}{ Setor indutor } \\
\hline & Agropecuária & Indústria & Serviços \\
\hline Agropecuária & $6,73 \%$ & $8,24 \%$ & $-3,73 \%$ \\
\hline Indústria extrativa mineral & $0,79 \%$ & $7,67 \%$ & $4,47 \%$ \\
\hline Indústria de transformação & $5,14 \%$ & $-12,59 \%$ & $-1,75 \%$ \\
\hline $\begin{array}{l}\text { Produção e distribuição de } \\
\text { eletricidade, gás e água }\end{array}$ & $0,45 \%$ & $5,20 \%$ & $11,45 \%$ \\
\hline Construção Civil & $0,06 \%$ & $-3,51 \%$ & $-1,32 \%$ \\
\hline Comércio & $1,29 \%$ & $3,48 \%$ & $1,03 \%$ \\
\hline Transportes & $0,18 \%$ & $1,92 \%$ & $10,40 \%$ \\
\hline Comunicações & $0,27 \%$ & $-2,71 \%$ & $70,48 \%$ \\
\hline (continua) & & & \\
\hline
\end{tabular}




\begin{tabular}{l|ccc}
\hline (Continuação) & \multicolumn{3}{c}{ VALOR ADICIONADO } \\
\cline { 2 - 4 } \multicolumn{1}{c}{ Atividade sob efeito } & \multicolumn{3}{c}{ Setor indutor } \\
\cline { 2 - 4 } & Agropecuária & Indústria & Serviços \\
\hline Instituições Financeiras & $0,28 \%$ & $5,28 \%$ & $3,37 \%$ \\
Serviços Prestados à Familía & $0,04 \%$ & $0,02 \%$ & $-3,25 \%$ \\
Serviços Prestados à Empresa & $0,33 \%$ & $10,51 \%$ & $-10,82 \%$ \\
Aluguel de Imóveis & $0,27 \%$ & $3,55 \%$ & $-1,73 \%$ \\
Administração Pública & $-0,03 \%$ & $-0,91 \%$ & $-1,87 \%$ \\
Serviços Privados não-mercantis: & & & \\
Educação; Saúde e serviços sociais; & $-0,14 \%$ & $-4,76 \%$ & $-6,86 \%$ \\
Outros serviços coletivos, sociais e & & & \\
pessoais & & & \\
\hline
\end{tabular}

Fonte: elaborado pela autora, com base nos dados do NEREUS.

TABELA 5 - DECOMPOSIÇÃO DESAGREGADA DO EFEITO TECNOLÓGICO (CONTINUAÇÃO)

\begin{tabular}{l|c|c|c}
\hline \multirow{2}{*}{\multicolumn{1}{c}{ Atividade sob efeito }} & \multicolumn{3}{|c}{ VBP } \\
\cline { 2 - 4 } & \multicolumn{3}{|c}{ Setor indutor } \\
\cline { 2 - 4 } & Agropecuária & Agropecuária & Agropecuária \\
\hline Agropecuária & $4,32 \%$ & $5,71 \%$ & $-2,38 \%$ \\
Indústria extrativa mineral & $0,88 \%$ & $8,28 \%$ & $4,64 \%$ \\
Indústria de transformação & $3,07 \%$ & $-8,38 \%$ & $0,96 \%$ \\
Produção e distribuição de & $0,38 \%$ & $4,47 \%$ & $9,76 \%$ \\
eletricidade, gás e água & $0,06 \%$ & $-3,67 \%$ & $-1,38 \%$ \\
Construção Civil & $1,10 \%$ & $3,01 \%$ & $0,89 \%$ \\
Comércio & $0,10 \%$ & $1,18 \%$ & $5,82 \%$ \\
Transportes & $0,15 \%$ & $-1,51 \%$ & $38,80 \%$ \\
Comunicações & $0,33 \%$ & $6,27 \%$ & $4,00 \%$ \\
Instituições Financeiras & $0,05 \%$ & $0,02 \%$ & $-3,97 \%$ \\
Serviços Prestados à & & & \\
Familía & $0,34 \%$ & $10,78 \%$ & $-11,10 \%$ \\
Serviços Prestados à & $0,24 \%$ & $3,25 \%$ & $-1,58 \%$ \\
Empresa & $-0,02 \%$ & $-0,81 \%$ & $-1,67 \%$ \\
Aluguel de Imóveis & & & \\
Administração Pública & \multicolumn{3}{|c}{} \\
\hline Continua) & & & \\
\hline
\end{tabular}




\begin{tabular}{|c|c|c|c|}
\hline \multicolumn{4}{|l|}{ (Continuação) } \\
\hline \multirow{3}{*}{ Atividade sob efeito } & \multirow{2}{*}{\multicolumn{3}{|c|}{$\begin{array}{c}\text { VBP } \\
\text { Setor indutor }\end{array}$}} \\
\hline & & & \\
\hline & Agropecuária & Agropecuária & Agropecuária \\
\hline $\begin{array}{l}\text { Serviços Privados não- } \\
\text { mercantis: Educação; Saúde } \\
\text { e serviços sociais; Outros } \\
\text { serviços coletivos, sociais e } \\
\text { pessoais }\end{array}$ & $-0,16 \%$ & $-5,47 \%$ & $-7,89 \%$ \\
\hline
\end{tabular}

Fonte: elaborado pela autora, com base nos dados do NEREUS.

\subsection{Decomposição da Mudança na Demanda Final}

A Tabela 6 permite identificar a parcela do crescimento do emprego, valor adicionado e VBP determinada por cada efeito da demanda final (nível, mix e composição). O aumento do nível da demanda final foi o principal responsável pelos crescimentos do emprego, do valor adicionado e do VBP dos três setores (agropecuária, indústria e serviços).

TABELA 6 - DECOMPOSIÇÃO DOS EFEITOS DA DEMANDA FINAL

\begin{tabular}{|c|c|c|c|c|}
\hline & \multirow{2}{*}{$\begin{array}{c}\text { Efeitos } \\
\text { Demanda } \\
\text { Final } \\
\end{array}$} & \multicolumn{3}{|c|}{ Setor } \\
\hline & & Agropecuária & Indústria & Serviços \\
\hline \multirow{3}{*}{ EMPREGO } & Nível & $-433,98 \%$ & $170,83 \%$ & $128,46 \%$ \\
\hline & Mix & $12,47 \%$ & $-25,84 \%$ & $9,92 \%$ \\
\hline & Composição & $-47,92 \%$ & $-3,92 \%$ & $-22,00 \%$ \\
\hline \multirow{3}{*}{$\begin{array}{l}\text { VALOR } \\
\text { ADICIONADO }\end{array}$} & Nível & $131,45 \%$ & $122,78 \%$ & $101,34 \%$ \\
\hline & Mix & $-5,77 \%$ & $-10,49 \%$ & $4,69 \%$ \\
\hline & Composição & $14,70 \%$ & $-38,82 \%$ & $-73,58 \%$ \\
\hline \multirow{3}{*}{$\begin{array}{l}\text { VALOR } \\
\text { BRUTO DE } \\
\text { PRODUÇÃO }\end{array}$} & Nível & $86,92 \%$ & $102,77 \%$ & $91,09 \%$ \\
\hline & Mix & $-4,33 \%$ & $-7,60 \%$ & $6,91 \%$ \\
\hline & Composição & $9,77 \%$ & $-71,52 \%$ & $-47,37 \%$ \\
\hline
\end{tabular}

Fonte: elaborado pela autora, com base nos dados do NEREUS.

Foi visto que o emprego da agropecuária sofreu redução no período pela Tabela 1 e que as mudanças tecnológicas ocorridas no setor de serviços cola- 
boraram para essa redução pela Tabela 4. Agora, pela Tabela 6, percebe-se que as mudanças no nível e na composição da demanda final no período elevaram o emprego desse setor, em 433,98\% e 47,92\%, respectivamente. Já as mudanças no mix da demanda final foram as que colaboraram para a redução do emprego na agropecuária, em 12,47\%.

Pela Tabela 6, o efeito mix e composição da demanda final reduziram os crescimentos do emprego, valor adicionado e VBP do setor industrial. Para o setor de serviços, o efeito mix aumentou o crescimento do emprego, valor adicionado e VBP, enquanto o efeito composição reduziu esses crescimentos.

O item referente à demanda final no Sistema de Contas Nacionais é constituído pela soma de seis componentes: Exportações, Consumo da Administração Pública, Consumo das ISFLSFs, Consumo das Famílias, FBCF e Variação de Estoque. A Tabela 7 mostra o crescimento percentual do nível de cada um destes agregados entre 1995 e 2009. Nota-se que as exportações apresentaram maior crescimento no período. Então, como o nível da demanda agregada foi o principal responsável pelos crescimentos dos setores, o crescimento das exportações foi o principal responsável para explicar estes crescimentos.

TABELA 7 - CRESCIMENTO DOS AGREGADOS MACROECONÔMICOS DA DEMANDA FINAL ENTRE 1995 E 2009

\begin{tabular}{lc}
\hline Agregados macroeconômicos & Crescimento \\
\hline Exportações & $137,76 \%$ \\
Consumo da Administração Pública & $52,46 \%$ \\
Consumo das ISLSFs & $49,46 \%$ \\
Consumo das famílias & $68,16 \%$ \\
FBCF & $60,42 \%$ \\
Variação de Estoque & $-121,01 \%$ \\
\hline Total & $68,00 \%$ \\
\hline
\end{tabular}

Fonte: elaborado pela autora, com base nos dados do NEREUS.

A Tabela 8 mostra o mix da demanda final no período, mudanças setoriais, dentro do mesmo agregado macroeconômico. Para a agropecuária e a indústria o efeito mix reduziu os crescimentos do emprego, valor adicionado e VBP, já para serviços esse efeito aumentou os crescimentos (Tabela 6). As mudanças no consumo das famílias e na FBCF explicam esse fato. O consumo das famílias e a FBCF sofreram reduções de suas parcelas na demanda final de 2009 em relação à suas parcelas na demanda final de 1995 para a agropecuária e indústria, enquanto para serviços houve aumentos das parcelas desses agregados. 
FIGUEIREDO, H. L. de; OLIVEIRA, M. A. S. Análise de Decomposição Estrutural para a Economia brasileira ...

TABELA 8- MIX DA DEMANDA FINAL EM 1995 E 2009

\begin{tabular}{|c|c|c|c|c|c|c|}
\hline \multirow{3}{*}{$\begin{array}{l}\text { Agregados } \\
\text { Macroeconômicos }\end{array}$} & \multicolumn{6}{|c|}{ Mix } \\
\hline & \multicolumn{2}{|c|}{ Agropecuária } & \multicolumn{2}{|c|}{ Indústria } & \multicolumn{2}{|c|}{ Serviços } \\
\hline & 1995 & 2009 & 1995 & 2009 & 1995 & 2009 \\
\hline $\begin{array}{l}\text { Exportação de } \\
\text { Bens e Serviços }\end{array}$ & $0,39 \%$ & $0,99 \%$ & $5,09 \%$ & $6,61 \%$ & $1,85 \%$ & $2,78 \%$ \\
\hline $\begin{array}{l}\text { Consumo da Adm. } \\
\text { Pública }\end{array}$ & $0,00 \%$ & $0,00 \%$ & $0,00 \%$ & $0,09 \%$ & $23,40 \%$ & $21,15 \%$ \\
\hline $\begin{array}{l}\text { Consumo das } \\
\text { ISFLSF }\end{array}$ & $0,00 \%$ & $0,00 \%$ & $0,00 \%$ & $0,00 \%$ & $1,34 \%$ & $1,20 \%$ \\
\hline $\begin{array}{l}\text { Consumo das } \\
\text { famílias }\end{array}$ & $2,16 \%$ & $1,89 \%$ & $19,64 \%$ & $17,70 \%$ & $30,43 \%$ & $32,69 \%$ \\
\hline $\mathrm{FBCF}$ & $0,46 \%$ & $0,44 \%$ & $13,50 \%$ & $12,71 \%$ & $1,93 \%$ & $2,03 \%$ \\
\hline $\begin{array}{l}\text { Variação de } \\
\text { Estoque }\end{array}$ & $-0,04 \%$ & $-0,03 \%$ & $-0,13 \%$ & $-0,20 \%$ & $-0,03 \%$ & $-0,04 \%$ \\
\hline $\begin{array}{l}\text { Total (Demanda } \\
\text { Final) }\end{array}$ & $2,96 \%$ & $3,28 \%$ & $38,11 \%$ & $36,91 \%$ & $58,93 \%$ & $59,80 \%$ \\
\hline
\end{tabular}

Fonte: elaborado pela autora, com base nos dados do NEREUS.

Finalmente, a Tabela 9 exibe os resultados para o efeito composição da demanda final. $\mathrm{O}$ efeito composição mostra mudanças entre os agregados macroeconômicos da demanda final. Pela Tabela 6, vimos que o efeito composição aumentou o crescimento do emprego, valor adicionado e VBP para a agropecuária e reduziu esses crescimentos para indústria e serviços. Pela Tabela 9, os aumentos dos pesos das exportações e do consumo das famílias na demanda final de 2009 comparada a de 1995 contribuíram para os crescimentos do emprego, valor adicionado e VBP da agropecuária. Enquanto as reduções dos pesos do consumo da administração pública, do consumo das ISFLSF e da FBCF contribuíram para a redução dos crescimentos do emprego, valor adicionado e VBP da indústria e serviços. 
TABELA 9- COMPOSIÇÃO DA DEMANDA FINAL EM 1995 E 2009)

\begin{tabular}{l|c|c}
\hline \multirow{2}{*}{$\begin{array}{l}\text { Agregados } \\
\text { Macroeconômicos }\end{array}$} & 1995 & 2009 \\
\cline { 2 - 3 } $\begin{array}{l}\text { Exportação de Bens e } \\
\text { Serviços }\end{array}$ & $7,33 \%$ & $10,38 \%$ \\
Consumo da Adm. & $23,40 \%$ & $21,24 \%$ \\
Pública & $1,34 \%$ & $1,20 \%$ \\
Consumo das ISFLSF & $52,22 \%$ & $52,27 \%$ \\
Consumo das famílias & $15,90 \%$ & $15,18 \%$ \\
FBCF & $-0,20 \%$ & $-0,26 \%$ \\
Variação de Estoque & $100,00 \%$ & $100,00 \%$ \\
\hline Total (Demanda Final) & & \\
\hline
\end{tabular}

Fonte: elaborado pela autora, com base nos dados do NEREUS.

Tanto o efeito mix como o efeito composição apontaram a redução da parcela da FBCF, comparando a demanda final de 2009 com a de 1995, como um fator comum explicativo para a redução do crescimento do emprego, valor adicionado e VBP da indústria. Esse aspecto vai de encontro aos estudos de Messa (2012) e Rowthorn e Ramaswamy (1999).

Messa (2012) indicou em seu artigo que para se incentivar o setor industrial deve-se procurar o provimento de incentivos à $\mathrm{FBCF}$, em detrimento do consumo da administração pública. O autor salientou que a FBCF é importante para as indústrias de transformação e de construção e que os gastos do governo impactaram significativamente apenas os serviços. No restante da economia, seu papel mostrou-se reduzido. Rowthorn e Ramaswamy (1999) detectaram correlações positivas entre o nível de FBCF e a participação da indústria no emprego e na produção.

\section{Considerações Finais}

Conforme abordado anteriormente, a abertura comercial e financeira da economia brasileira na década de 1990 intensificou a necessidade de reestruturação dos setores produtivos da economia. Diante de um novo cenário, em que se passa a enfrentar a concorrência externa abertamente, houve a perda de participação do setor industrial no valor adicionado e no emprego e o crescimento do setor de serviços. $\mathrm{O}$ assunto tem provocado um intenso debate acerca de suas consequências. Este artigo procurou contribuir com este debate, revelando, dentre outros resultados, um exponencial crescimento do 
setor de serviços no período, especificadamente para comunicações e serviços prestados à família.

Entre todas as atividades, comunicações apresentou o maior crescimento do emprego e do VBP explicado principalmente pelo aumento da demanda final, mas também pela mudança tecnológica do setor de serviços. Serviços prestados à família refere-se à atividades de alojamento, alimentação e outros serviços; apresentou o maior crescimento do valor adicionado no período explicado pela mudança na demanda final e, em segundo lugar, pelo coeficiente direto de valor adicionado.

O setor agropecuário apresentou redução do emprego no período, explicada pela elevação da produtividade do trabalho. Entretanto apresentou o maior crescimento do VBP causado, principalmente, pela elevação do nível e pela mudança na composição da demanda final. O crescimento dos agregados da demanda final e o aumento do peso das exportações e do consumo das famílias na demanda final de 2009 comparada a de 1995 contribuíram para esse resultado.

Já em relação ao setor industrial, ele apresentou crescimento do emprego e do valor adicionado superior ao da agropecuária, porém inferior ao setor de serviços. Entre as atividades da indústria o maior crescimento do valor adicionado e do VBP foi para a Indústria Extrativa Mineral e o maior crescimento do emprego foi para Construção Civil. A Indústria de Transformação sofreu redução no crescimento do emprego, valor adicionado e VBP pela mudança tecnológica ocorrida no próprio setor industrial.

O aumento do nível da demanda final foi o principal responsável pelo crescimento do emprego, valor adicionado e VBP, especialmente o aumento das exportações de bens e serviços e do consumo das famílias no período.

Então, o padrão de crescimento dos setores da economia brasileira entre 1995 e 2009 se caracterizou pelo aumento da produtividade do trabalho do setor agropecuário, o que liberou mão-de-obra para os setores industriais e de serviços. O setor industrial apresentou crescimentos, mesmo que inferiores ao de setor de serviços, puxados pela Indústria Extrativa Mineral e pela Construção Civil. É importante notar que as mudanças tecnológicas ocorridas no setor industrial reduziram o crescimento de importantes atividades, como a indústria de transformação e a construção civil. Ao mesmo tempo essas mudanças tecnológicas ocorridas no setor industrial colaboraram para o crescimento do setor de serviços.

Não foi encontrada uma queda do crescimento do setor industrial e nem de suas atividades em termos do emprego, valor adicionado e VBP. Mas tendo em vista que o aumento do nível da demanda final foi o principal responsável pelo crescimento desses agregados no setor industrial, e que para a Indústria de Transformação o nível da demanda final foi o único responsável pelos 
crescimentos, analisar os outros efeitos (tecnológico, mix e composição) é importante para tentar visualizar medidas compensatórias para a indústria.

Para a Indústria de Transformação, o efeito tecnológico mostrou que as mudanças ocorridas no setor industrial reduziram o crescimento do emprego, valor adicionado e do VBP. Ou seja, houve um enfraquecimento dos elos entre os setores da indústria de transformação, o que pode ter ocorrido com substituições de insumos nacionais por importados, mudanças no mix de produtos (com a adoção de novos substitutos ou de insumos complementares no processo produtivo), mudança dos preços relativos e mudanças nos padrões de troca da indústria.

Ainda para esse setor, o efeito mix da demanda final apontou que a redução das parcelas da FBCF e do consumo das famílias, comparando a demanda final de 2009 com a de 1995, contribuiu para as reduções dos crescimentos do emprego, valor adicionado e VBP. Já o efeito composição aponta para as reduções do peso do consumo da administração pública, do consumo das ISFLSF e da FBCF.

É possível concluir, por este artigo que, caso as medidas compensatórias ao crescimento da indústria de transformação sejam de interesse do formulador de políticas públicas, deve-se incentivar o fortalecimento dos elos industriais e a FBCF, que influenciará o efeito mix e o efeito composição da demanda final positivamente elevando o crescimento da indústria.

\section{Referências}

Araújo, E. L. (2010), "Efeitos das variáveis macroeconômicas sobre o desempenho da indústria de transformação brasileira: uma análise do período 1994-2009”, paper presented at the $13^{\circ}$ Encontro Regional de Economia da Região SulANPEC, Porto Alegre.

Carvalho, L. and Kupfer, D. (2011) "Diversificação ou especialização: uma análise do processo de mudança estrutural da indústria brasileira”, Revista de Economia Política, São Paulo, 31 (4): 618-637.

Cimoli, M. (2005) "Structural heterogeneity, technological asymmetries and growth in Latin America”, ECLAC's Publications 35: 1-162.

Dietzembacher, E. and Los, B. (1998) "Structural decomposition techniques: sense and sensitive", Economic Systems Research, Abingdon, 10 (4): 307-323.

Franke, R. and Kalmbach, P. (2005) "Structural change in the manufacturing sector and its impact on business-related services: an input-output study for Germany", Structural Change and Economic Dynamics, Amsterdam, 16: 467-488.

Guilhoto, J. J. M. and Sesso Filho, U. A. (2010) "Estimação da matriz insumo-produto utilizando dados preliminares das contas nacionais: aplicação e análise 
de indicadores econômicos para o Brasil em 2005”, Economia e Tecnologia, [S.1.], 23: 53-62.

Guilhoto, J. J. M., Hewings, G. J. D., Sonis, M. and Jiemin, G. (2001) "Economic structural change over time Brazil and the United States compared”, Journal of Policy Modeling, New York, 23: 703-711.

Hirschman, A. (1958), The strategy of economic development, New Haven: Vale University Press.

Instituto Brasileiro de Geografia e Estatística. (2014) "Sistema de Contas Nacionais: tabelas recursos e usos de 1996 a 2009”, available at: http://www.ibge.gov.br (accessed 2 July 2014).

Kuznets, S. (1957) "Quantitative aspects of the economic growth of nations, Part II", Economic Development and Cultural Change, 5 (4): 1-111.

Linden, J. A. and Dietzenbacher, E. (2000) “The Determinants of Structural Change in the European Union: A New Application of RAS” Environment and Planning, London, 32 (12): 2205-2229.

Messa, A. (2012) "Mudanças estruturais na economia brasileira ao longo da década de 200o", Instituto de Pesquisa Econômica Aplicada, Brasília (Texto para discussão, n. 1770).

Miernyk, W. H. (1974) Elementos de análise do insumo-produto, São Paulo: Atlas.

Miller, R. E and Blair, P. D. (2009) Input-output analysis: foundations and extensions, New Jersey: Prentice-Hall.

Oreiro, J. L. and Feijó, C. A. (2010) "Desindustrialização: conceituação, causas, efeitos e o caso brasileiro", Revista de Economia Política, São Paulo, 30 (2): 100-115.

Prebisch, R. (1981) Capitalismo Periférico: Crisis y Transformación, México: Fondo de Cultura Económica.

Ramos, R. L. O. (1996) "Mudanças estruturais nas matrizes de insumo-produto: Brasil - 1980/85”, Revista Política e Planejamento Econômico, Rio de Janeiro, 26 (1): 93-118.

Rodrik, D. (2013). "Structural change, fundamentals and growth: an overview", United States: Institute for Advanced Study.

Rowthorn, R. and Ramaswamy, R. (1999) "Growth, trade and deindustrialization", International Monetary Fund, IMF Staff Papers, Washington, 46 (1): 18-41.

Sarti, M. and Laplane, M. (2002) "Investimento direto estrangeiro e a internacionalização da economia brasileira nos anos 1990", Economia e Sociedade, Campinas, 18: 63-94.

Schuschny, A. R. (2005) "Tópicos sobre el modelo de insumo-producto: teoria y aplicaciones", United Nations Publications, n. 37.

Suttcliffe, R. B. (1971) “Industry and Underdevelopment”, London: Addison-Wesley Publishing Company. 